\title{
A Four-Stokes-Parameter Spectral Line Polarimeter at the Caltech Submillimeter Observatory
}

\author{
Talayeh Hezareh $^{1}$, \\ hezareh@astro.uwo.ca \\ Martin Houde ${ }^{1}$
}

\begin{abstract}
We designed and built a new Four-Stokes-Parameter spectral line Polarimeter (FSPPol) for the Caltech Submillimeter Observatory (CSO). The simple design of FSPPol does not include any mirrors or optical components to redirect or re-image the radiation beam and simply transmits the beam to the receiver through its retarder plates. FSPPol is currently optimized for observation in the $200-260 \mathrm{GHz}$ range and measures all four Stokes parameters, $I, Q, U$, and $V$. The very low level of instrument polarization makes it possible to obtain reliable measurements of the Goldreich-Kylafis effect in molecular spectral lines. Accordingly, we measured a polarization fraction of a few percent in the spectral line wings of ${ }^{12} \mathrm{CO}(J=2 \rightarrow 1)$ in Orion $\mathrm{KL} / \mathrm{IRc} 2$, which is consistent with previous observations. We also used FSPPol to study the Zeeman effect in the $N=2 \rightarrow 1$ transition of $\mathrm{CN}$ in $\mathrm{DR} 21(\mathrm{OH})$ for the first time. At this point we cannot report a Zeeman detection, but more observations are ongoing.
\end{abstract}

Subject headings: Instrumentation: Polarimeters-ISM: Clouds-ISM: Magnetic FieldsSubmillimeter

\section{Introduction}

Astronomical polarimetry is a powerful tool for studying the characteristics of the interstellar medium, from the large scale galactic magnetic fields to the gravitational collapse of molecular cloud cores. The understanding of the physical phenomena responsible for polarized interstellar emission provides valuable information about the underlying astrophysical processes, an important example being the formation and evolution of stars. Since the 1990s, polarimetry techniques and instruments have been developed for a wide range of wavelengths, from optical (e.g., Magalhaes et al. 1996; Wiktorowicz \& Matthews 2008) to the submillimeter and radio regimes (e.g., Platt et al. 1991; Hildebrand et al. 1997; Shinnaga \& Tsuboi 1999; Greaves et al. 2003; Li et al. 2008; Thum et al. 2008; Hildebrand et al. 2009; Houde et al. 2009; Heiles 2001; Heiles et al. 2001a,b).

\footnotetext{
${ }^{1}$ The Department of Physics and Astronomy, The University of Western Ontario, London, Ontario, Canada N6A $3 \mathrm{~K} 7$
} 
One of the important applications of astronomical polarimetry lies in the study of the effect of magnetic fields on the early stages of star formation. Hence, it is important to characterize the interstellar magnetic fields around star-forming regions. Indeed, it is possible to obtain the orientation and strength of the magnetic fields observationally, as they leave their signature on the emission of the interstellar dust grains and molecules. For example, the dust continuum radiation becomes linearly polarized in a magnetic field (Hildebrand et al. 1999). At submillimeter wavelengths this polarization is perpendicular to the field lines and a polarimetry map of dust continuum emission will therefore reveal the plane-of-the-sky orientation of the magnetic field. Furthermore, in the presence of anisotropic radiation, magnetic fields cause the emission from gas molecules to also be linearly polarized by a few percent (Goldreich \& Kylafis 1981). It is also possible to determine whether this polarization is aligned parallel or perpendicular to the plane-of-the-sky component of the ambient magnetic field (Deguchi \& Watson 1984). Finally, the only way to directly measure the strength of interstellar magnetic fields is through Zeeman line-broadening measurements. More precisely, the line-of-sight component of the field is obtained by measuring the circular polarization in the emission of the Zeeman components of molecular spectral lines (Crutcher et al. 1993, 1999).

We describe the Four-Stokes-Parameter spectral line Polarimeter (FSPPol) we recently designed and successfully commissioned at the Caltech Submillimeter Observatory (CSO) in November 2008 in $\S 2$. We present linear polarization measurements of ${ }^{12} \mathrm{CO}(J=2 \rightarrow 1)$ in Orion KL/IRc2 in $\S 3$ and preliminary Zeeman observations of the $N=2 \rightarrow 1$ transition of $\mathrm{CN}$ in DR21(OH) in $\S 4$. We end with a summary in $\S 5$.

\section{Instrument Description}

The design of FSPPol is based on single beam polarimetry, as the current heterodyne receivers at the CSO can measure only one linear polarization state at one position at a time. The simple construction of the polarimeter enabled us to mount the instrument inside the elevation tube that traces much of the optical path from the tertiary mirror behind the telescope dish to the Nasmyth focus where the $200-300 \mathrm{GHz}$ receiver is located. A schematic diagram of FSPPol installed in the elevation tube is shown in Figure 1. The polarimeter is mounted on a bracket attached to the walls of the tube and its location with respect to the tertiary mirror and the receiver is depicted with the schematic diagram of the CSO telescope in Figure 2. The third focus of the $230 \mathrm{GHz}$ telescope beam is virtual and located $1.07 \mathrm{~m}$ behind the tertiary mirror and has a waist of 35.9 $\mathrm{mm}$. We calculated the beam waist upon incidence on FSPPol to be $39.5 \mathrm{~mm}$. As illustrated in Figure 1, FSPPol is comprised of a half-wave plate (HWP) and a quarter-wave plate (QWP), each being $100 \mathrm{~mm}$ in diameter and optimized for observations at $226 \mathrm{GHz}$. The wave plates were manufactured by Meller Optics, Inc 1 and subsequently anti-reflection coated by QMC Instruments

\footnotetext{
${ }^{1} 120$ Corliss Street, Providence, RI 02904 USA
} 
Ltd2. More detailed specifications of the wave plates are listed in Table 1. These plates are installed in rotating rings mounted side by side on an aluminum translational stage that moves across the elevation tube, placing either wave plate in the path of the signal reflected from the tertiary mirror. The anti-reflection coating on the plates is efficient in eliminating standing waves along the optical path. Nevertheless, the mounting of the translational stage was adjusted in such a manner that the telescope beam impinged on the HWP or QWP at an incidence that is slightly off from normal (i.e., at most a few degrees) to avoid potential standing waves. The rotational and translational motions of these wave plates are precisely monitored and controlled by the instrument controlling software from the observatory's control room. The system temperature increases by about $8 \%$ when the HWP is placed in the path of the beam, and by about $6 \%$ with the QWP in the beam.

We aligned the optical axes of the wave plates in relation with the polarization axis of the receiver by measuring the intensity from a cold load, located at the entrance of FSPPol, while rotating the wave plates over a wide range of angles. We previously placed a wire grid with its polarizing state parallel to the receiver's axis between the wave plates and the cold load to maintain a specific incoming polarization state for testing purposes; this grid was removed for astronomical observations. It is straightforward to show that the location of a minimum in measured intensity with this set-up corresponds to an angle where either the fast or slow axis of a plate (HWP or QWP) is aligned with the receiver axis. This allows for a precise orientation of the HWP and QWP with the receiver axis (also see $\S 3$ ). It should also be noted that the slow axis is marked by the manufacturer on each plate, which we used as a further consistency check. A small circular cold load was also used to position the polarimeter within the elevation tube such that the telescope beam was centered on the surface of the HWP and QWP.

To test the instrument's accuracy, we observed the linear polarization in the $J=2 \rightarrow 1$ transition of ${ }^{12} \mathrm{CO}$ towards the Orion KL/IRc2 high mass star forming region. After successful linear polarization tests, we proceeded to perform Zeeman observations on the $N=2 \rightarrow 1$ transition of $\mathrm{CN}$ in DR21 $(\mathrm{OH})$ for the first time. The details of these observations are explained in the following sections.

\section{Linear Polarization Measurements}

We observed ${ }^{12} \mathrm{CO}(J=2 \rightarrow 1)$ at $230.54 \mathrm{GHz}$ in three different locations in Orion KL/IRc2 coincident with previous polarization observations by Girart et al. (2004), in order to assess the performance of FSPPol. These locations, defined in the equatorial coordinate system, were offset $\left(20^{\prime \prime}, 20^{\prime \prime}\right),\left(20^{\prime \prime},-20^{\prime \prime}\right)$ and $\left(-20^{\prime \prime},-20^{\prime \prime}\right)$ from the center of IRc2 $\left(\alpha=05^{\mathrm{h}} 35^{\mathrm{m}} 14.50^{\mathrm{s}}\right.$ and $\delta=$ $\left.-05^{\circ} 22^{\prime} 30.4^{\prime \prime}, J 2000.0\right)$. The observations were performed between September $27^{\text {th }}$ and October $6^{\text {th }}$ 2009 using the $200-300 \mathrm{GHz}$ receiver at the CSO, and the FFTS spectrometer with a bandwidth

\footnotetext{
${ }^{2}$ Cardiff University, School of Physics and Astronomy, The Parade, Cardiff CF24 3AA UK
} 
of $500 \mathrm{MHz}$ and a channel width of $61 \mathrm{kHz}$ that corresponds to a velocity resolution of about 0.08 $\mathrm{km} \mathrm{s}^{-1}$. The telescope efficiency was determined with scans on Jupiter and calculated to be $\simeq 65$ $\%$ for a beam width of $\simeq 32^{\prime \prime}(\mathrm{FWHM})$. During these observations the typical optical depth at 225 $\mathrm{GHz}$, as obtained with the $\mathrm{CSO}$ radiometer, was $\tau_{225} \approx 0.06$ and the typical system temperature with FSPPol in use was $T_{\text {sys }} \approx 370 \mathrm{~K}$.

Although the refractive indices for the ordinary and extraordinary rays vary significantly with frequency, the birefringence of crystal Quartz appears to be relatively unchanged over a wide band (Afsar 1987) even though there is some uncertainty in the literature over its value (0.048 for the frequencies we are concerned with; Marrone and Rao 2008). However, since the HWP was operated at approximately $230.5 \mathrm{GHz}$, i.e., more than $4 \mathrm{GHz}$ away from its design frequency, we characterized its performance at the aforementioned frequency by measuring the power from a cold load at different orientations of the HWP slow axis with respect to the polarization axis of the facility receiver at the CSO. Figure 3 shows the plot of the power at the receiver against different angles between the HWP and receiver axis. The power is measured in arbitrary units and the angles are displayed in degrees. A polarizing grid, with its polarization state parallel to the receiver axis, was placed between the cold load and the HWP to maintain a specific incoming polarization state. As seen in the figure the HWP response is very good at that frequency, although the power at successive minima is observed to slightly vary (Savini et al. 2009). Our model for the fit to the data in Figure 3 is a cosine function with the form

$$
I(\theta)=\frac{(\mathrm{CL}+\mathrm{HL})}{2}+\frac{(\mathrm{CL}-\mathrm{HL})}{2} \cos (4(\theta-\delta))
$$

where $I(\theta)$ is the power measured by the receiver at angle $\theta$ and $\delta$ determines the offset of the HWP slow axis from the orientation defining the polarization state of the receiver. CL is the power from the cold load and HL is the power due to emission from the receiver that reflects back on the wires of the polarizing grid; The HL signal incident on the HWP is therefore polarized perpendicular to the CL signal. It should also be noted that there is an equal contribution to CL and HL from receiver noise, although we cannot quantify it with these measurements alone. The fit values for CL, HL and $\delta$ are $722.2 \pm 2.1,1373.0 \pm 2.9$ and $1.45^{\circ} \pm 0.11^{\circ}$, respectively.

The polarization efficiency of FSPPol for linear polarization measurements was previously determined in November 2008 by taking scans on Saturn. We placed a wire grid at the entrance of FSPPol with its polarization state parallel to the receiver's axis to force a precise incoming polarization state, and observed Saturn for HWP angles $\theta=0^{\circ}, 90^{\circ}, 45^{\circ}$ and $135^{\circ}$, where $\theta$ is defined as the angle between the HWP slow axis and the receiver polarization axis. We performed a one-minute (on-source) integration for each measurement, with the system temperature being calibrated before every scan and the signal from Saturn being integrated over the whole available spectral bandwidth. Assuming that the wire grid is perfectly polarizing and that Saturn is unpolarized, we obtained a polarization efficiency of $\simeq 99 \%$. These measurements also allowed us to estimate the instrument polarization to be on the order of $\simeq 0.3 \%$. In view of this high polarization efficiency, we did not 
correct our Orion KL/IRc2 data for the efficiency and instrumental effects.

The polarimeter and the receiver are mounted in such a way that they rotate in elevation with the telescope and the polarization axis of the receiver on the sky, which is precisely oriented eastwest (parallel to the horizon) when FSPPol is not used, is preserved regardless of the pointing of the telescope since the CSO telescope has an alt-azimuth mount. Our aim for linear polarization measurements was to obtain the Stokes $Q$ and $U$ parameters in the reference frame of the sky, i.e., in the equatorial coordinate system, as well as the Stokes $I$. Therefore, the rotation of the object's orientation on the sky, or in other words the changes in its parallactic angle with time had to be considered. For this purpose, we obtained one-minute (on-source) long intensity measurements for different orientations of the HWP slow axis relative to the receiver polarization axis $\theta=\left(\gamma+\theta^{\prime}+\right.$ $\left.90^{\circ}\right) / 2$, where $\gamma$ is the parallactic angle defining the orientation of the object on the sky and $\theta^{\prime}$ is the angle at which we seek to measure the linear polarization state in the reference frame of the source (i.e., the frame that is rotated by $\gamma$ in the equatorial system; see Figure 4). These measurements are denoted by $I_{\theta^{\prime}}$. The instrument controlling software continuously obtained the updated value of the parallactic angle from the observatory's antenna computer during the on-source integration, and compared it to the initial value at the beginning of the integration. Once the change in $\gamma$ exceeded a predetermined threshold (i.e., $1^{\circ}$ ), the software commanded the antenna computer to stop the integration, rotated the HWP by the updated angle using the new value for $\gamma$, and the integration resumed. Our observations were performed in cycles of four measurements for $\theta^{\prime}=0^{\circ}, 90^{\circ}, 45^{\circ}$ and $135^{\circ}$. Similar to the observations on Saturn, we took one minute long scans for each measurement, and calibrated the system temperature before every scan. We adopted this conservative observing plan to minimize the effect of pointing or calibration errors in the polarization data. The telescope pointing was verified hourly with typical "five points" integrations on CRL865 $\left(\alpha=06^{\mathrm{h}} 03^{\mathrm{m}} 59.8^{\mathrm{s}}\right.$ and $\delta=07^{\circ} 25^{\prime} 51.4^{\prime \prime}$, J2000.0) as our reference star.

With the aforementioned definition for $I_{\theta^{\prime}}$ we have

$$
\begin{aligned}
I & =\frac{\left(I_{0^{\circ}}+I_{90^{\circ}}+I_{45^{\circ}}+I_{135^{\circ}}\right)}{2} \\
Q & =I_{0^{\circ}}-I_{90^{\circ}} \\
U & =I_{135^{\circ}}-I_{45^{\circ}},
\end{aligned}
$$

and

$$
\begin{aligned}
p & =\frac{\sqrt{Q^{2}+U^{2}}}{I} \\
\mathrm{PA} & =\frac{1}{2} \arctan \left(\frac{U}{Q}\right)
\end{aligned}
$$

for the polarization fraction and angle (measured from north increasing eastwards), respectively. 
The results from our measurements are shown in Figure 5, where the top graphs show the Stokes $I$ spectra (corrected for the beam efficiency), while the middle and bottom graphs are for the corresponding polarization fractions and angles, respectively. For $p$ and PA the data were binned using six adjacent velocity channels and only values for which $p \geq 3 \sigma_{p}$ are plotted, where $\sigma_{p}$ is the uncertainty in the polarization fraction.

Table 2 displays the average values for $p$ and PA in the blue and red wings of the CO spectra, as well as the small polarization fraction detected at the center of the lines. These values were obtained after calculating the average of the Stokes $I, Q$ and $U$ across the stated velocity ranges. The small polarization level in the center of the lines is probably the contribution from the instrumental polarization, as it is expected that the linear polarization due to the Goldreich-Kylafis effect will be greatly reduced where the optical depth is high (Goldreich \& Kylafis 1981). The values of PA are generally uniform across the spectral lines except for the center of the lines, where the relative contribution from the instrumental polarization is significant.

One potential problem in the linear polarization measurements is the presence of polarized sidelobes that introduce false polarization signals in the data (Forbrich et al. 2008). This is more likely to happen when the telescope is pointed towards the edge of an extended source for the purpose of polarimetry measurements in low intensity regions. This way, it is possible that strong emission from the core of the source falls on these polarized sidelobes and contaminates the data. We have yet to determine the potential contribution of polarized sidelobes to data obtained with FSPPol at the CSO, we cannot therefore comment on their significance for our results on Orion KL. We hope to do so during a future observing run.

Our polarization results are in general consistent with the findings of Girart et al. (2004) (see their Figure 1). The polarization fraction that we calculate in the spectral line wings of ${ }^{12} \mathrm{CO}$ is in good agreement with their results, and the difference between their values for polarization angles and ours (i.e., $10^{\circ}-20^{\circ}$ ) could solely be the result of different telescope beam sizes. This could also explain the slight differences in line profiles and intensities between their Stokes $I$ spectra and ours. For example the secondary peak at $\approx 12 \mathrm{~km} \mathrm{~s}^{-1}$ in the offset positions obtained by Girart et al. (2004) is not resolved in our spectral data.

\section{Zeeman Measurements of CN $(N=2 \rightarrow 1)$}

As mentioned earlier, measurement of the Zeeman effect in interstellar molecular spectral lines is the only direct way to obtain the strength of magnetic fields in molecular clouds. The Zeeman line-broadening in an interstellar spectral line profile is directly proportional to the strength of the ambient magnetic field, and can be studied by observing the signature of circular polarization in the line profile. To this date, Zeeman detections have been reported in the spectral lines of a few species, namely H I, OH, $\mathrm{H}_{2} \mathrm{O}, \mathrm{CH}_{3} \mathrm{OH}$ and CN (Sarma \& Momiian 2009; Troland et al. 2008; Falgarone et al. 2008; Sarma et al. 2001; Crutcher et al. 1999; Plante et al. 1995). The high critical 
density of CN makes this molecule suitable for studying magnetic fields in dense regions. Additionally, the rotational transition lines of $\mathrm{CN}$ contain several hyperfine components that have different Zeeman splitting coefficients. This makes it possible to distinguish between the true Zeeman effect and instrumental effects that produce artificial circular polarization in the data (Crutcher et al. 1996). Although the Zeeman effect in the $N=1 \rightarrow 0$ transition of CN has previously been detected (Falgarone et al. 2008; Crutcher et al. 1999), to the best of our knowledge it has never been attempted at $N=2 \rightarrow 1$. The higher critical density of the latter will make it possible to probe denser regions in molecular clouds and better establish observationally how the magnetic field strength scales with density.

There are nine strong hyperfine components in the $N=2 \rightarrow 1$ transition of CN. The frequencies, Zeeman coefficients and relative intensities of these lines are displayed in Table 3, Similar to the $N=1 \rightarrow 0$ transition previously observed in $\mathrm{DR} 21(\mathrm{OH})$, the $\mathrm{CN}(N=2 \rightarrow 1)$ lines are double

peaked, suggesting the existence of two velocity components (Crutcher et al. 1999). These two velocity components may be arising from different regions, and may exhibit different field strengths. It is possible to observe the nine hyperfine components simultaneously and fit the observed circular polarization data to the following expression for both velocity components (Crutcher et al. 1999)

$$
V_{i}=a\left(I_{i 1}+I_{i 2}\right)+b_{1}\left(\frac{d I_{i 1}}{d \nu}\right)+b_{2}\left(\frac{d I_{i 2}}{d \nu}\right)+c_{1}\left(Z_{i} \frac{d I_{i 1}}{d \nu}\right)+c_{2}\left(Z_{i} \frac{d I_{i 2}}{d \nu}\right)
$$

where $V_{i}$ is the total circular polarization for hyperfine line $i$. In the above equation, the different sources of polarization are expressed in separate terms. The first term represents the polarization contribution from the error in intensity calibrations in the two polarization modes, $a$, that adds a small image of the intensity spectrum, $I_{i 1}+I_{i 2}$, to the $V_{i}$ spectra. The second and third terms are polarization contributions from the beam squint effect represented by $b_{1}$ and $b_{2}$, that is caused when the right and left handed polarization measurements do not probe exactly the same region, for which a non-zero velocity gradient exists in its vicinity (Crutcher et al. 1993). This effect is brought about by pointing errors of the telescope, and also in some cases by mechanical deformations in the antenna. The last two terms are the true Zeeman signals for each velocity component of the spectrum with $c_{1}$ and $c_{2}$ representing the strength of the line-of-sight component of the magnetic fields $B_{\operatorname{los} 1} / 2$ and $B_{\operatorname{los} 2} / 2$, respectively, for each velocity component, and $Z_{i}$ being the Zeeman coefficient for hyperfine line $i$. This way, the real Zeeman effect is separated from instrumental effects by determining the above fitting parameters.

\subsection{Observations}

We observed $\mathrm{CN}(N=2 \rightarrow 1)$ at $226.8 \mathrm{GHz}$ in $\mathrm{DR} 21(\mathrm{OH})\left(\alpha=20^{\mathrm{h}} 39^{\mathrm{m}} 01^{\mathrm{s}}\right.$ and $\delta=$ $42^{\circ} 22^{\prime} 37.7^{\prime \prime}$, J2000.0) during July $8^{\text {th }}$ to $13^{\text {th }}$ and September $27^{\text {th }}$ to October $6^{\text {th }} 2009$, using the FFTS spectrometer with bandwidth of $500 \mathrm{MHz}$ and channel resolution of $61 \mathrm{kHz}$. The telescope efficiency was determined with scans on Saturn and Jupiter and calculated to be $\simeq 65 \%$ for a beam 
width of $\simeq 32^{\prime \prime}$ (FWHM). We verified the telescope pointing on an approximately hourly rate by performing ${ }^{12} \mathrm{CO}(J=2 \rightarrow 1)$ scans on $\chi$ Cygni $\left(\alpha=19^{\mathrm{h}} 50^{\mathrm{m}} 33.8^{\mathrm{s}}\right.$ and $\delta=32^{\circ} 54^{\prime} 53.2^{\prime \prime}$, J2000.0) as our reference star. Our first observing session suffered from issues with an unstable receiver and mediocre skies. The average system temperature at that time was about $500 \mathrm{~K}$ and the typical $\tau_{225}$ was $\approx 0.12$. The circular polarization observations were performed by taking scans of $\mathrm{CN}$ $(N=2 \rightarrow 1)$ in $\mathrm{DR} 21(\mathrm{OH})$ with the QWP slow axis rotated by $+45^{\circ}$ and $-45^{\circ}$ with respect to the receiver's polarization axis. With our instrument set-up, our one minute on-source integrations at $+45^{\circ}$ project the right-handed circular polarization $\left(I_{R C P}\right)$ emission (on the sky) on the receiver's axis, while similar integrations at $-45^{\circ}$ probe the corresponding left-handed circular polarization $\left(I_{L C P}\right)$ emission. The Stokes $I$ and $V$ are obtained from $I=I_{L C P}+I_{R C P}$ and $V=I_{L C P}-I_{R C P}$. As

before, this observing strategy was chosen in order to mitigate any potential calibration or pointing errors. The system temperature was calibrated before every scan. We have so far obtained a total on-source integration time of 553 minutes, with an average system temperature of $450 \mathrm{~K}$.

Figure 6 shows the Stokes $I$ spectrum for the $\mathrm{CN}(N=2 \rightarrow 1)$ hyperfine lines that are labeled according to the order given in Table 3 with the line temperatures corrected for the telescope beam efficiency. There are two further hyperfine lines at $\simeq-45 \mathrm{~km} \mathrm{~s}^{-1}$ and $\simeq 200 \mathrm{~km} \mathrm{~s}^{-1}$ that are weak relative to the labeled lines and therefore not included in the study. Observing the $N=2 \rightarrow 1$ transition of $\mathrm{CN}$ brings more complications compared to the $N=1 \rightarrow 0$ transition. For example, there are three lines in Table 3 that are labeled 5 because they are heavily blended and appear as a single broad line in the observed spectrum, as seen in Figure 6. Furthermore, due to the double peaked feature of the $\mathrm{CN}$ lines, the other hyperfine lines with small frequency separations (i.e., lines 2 and 3 and lines 6 and 7) are blended together as well, although to a lesser level.

An inspection of the ratio of line temperatures reveals that our $\mathrm{CN}$ lines are not consistent with the LTE assumption. The spacing between the two velocity components of every hyperfine line varies with the line strength, and the rotational diagram for the $N=2 \rightarrow 1$ lines produced a negative excitation temperature, suggesting that the lines are affected by self absorption. A more complete spectral line analysis will be presented in a forth-coming paper.

\subsection{Preliminary Analysis \& Discussion}

The following is only a preliminary analysis on the data we have gathered so far, as more data will be obtained in upcoming observing runs, and a more thorough discussion will be presented then.

Since the CN hyperfine lines in DR21(OH) are double peaked, we fitted two to three Gaussian profiles to each line depending on its shape. In the fitting procedure, the line widths of the Gaussian profiles within each velocity component of a hyperfine line profile were kept the same. For the three blended hyperfine components of line 5 (in Figure 6), we fitted one Gaussian profile to each component and fixed their relative frequencies according to the information given in Table 3 , Each 
hyperfine component of the other pairs of blended lines (lines 2 and 3 and lines 6 and 7) were treated as single lines, such that Gaussian profiles incorporating both lines were fitted to the blended line profiles. Once the fitting parameters for all the Gaussian profiles were obtained, the blended lines could thus be separated by subtracting the Gaussian fit of one line from the spectrum of the pair. The Gaussian fits for the Stokes $I_{i}=I_{i 1}+I_{i 2}$ for both velocity components of every hyperfine line $i$, together with their derivatives with respect to frequency and corresponding Zeeman coefficients were simultaneously fitted to the Stokes $V$ spectrum using Equation (4). The resulting fitting parameters were $a=-0.0015 \pm 0.0007, b_{1}=1.24 \pm 1.13 \mathrm{kHz}, b_{2}=1.28 \pm 1.47 \mathrm{kHz}, c_{1}=-0.5 \pm 1.3$ $\mathrm{mG}$ and $c_{2}=-2.5 \pm 2.0 \mathrm{mG}$. Although we have not obtained a Zeeman detection, the instrumental polarization contribution is seen to be very small for $a$ and kept to reasonable levels for $b_{1}$ and $b_{2}$ (i.e., of the same order as the expected values for $c_{1}$ and $c_{2}$ ).

Since it is not easy to distinguish the Zeeman signal in the $V$ spectra of individual lines from the noise level, Crutcher et al. (1999) produced an averaged sum of the $N=1 \rightarrow 0$ hyperfine lines with strong Zeeman coefficients to display the Zeeman fit for their detection. In the case of the $N=2 \rightarrow 1$ transition, not all hyperfine lines have the same sign for their Zeeman coefficients and therefore it is only possible to average together the lines with same coefficient signs. The top panel of Figure 7 shows the weighted average Stokes $\bar{I}$ for lines 1, 2 and 4 that have negative Zeeman coefficients, with the weights being the relative sensitivity to the magnetic field, i.e., $\mid Z_{i} \times$ R. $_{i} \mid$ given in Table 3 , All the hyperfine lines are centered on the $V_{L S R}$ of the source, i.e., $-3 \mathrm{~km} \mathrm{~s}^{-1}$ and in order to remove the contamination of line 3 from line 2, the Gaussian fit for line 3 was subtracted from the blended spectrum. A similar procedure was performed for the averaged $V$ spectrum of the aforementioned lines, which is displayed in the lower panel of Figure 7 with the instrumental effects removed. The bold line is the Zeeman fit to the average $V$ data, expressed by $\bar{V}=\bar{Z}\left(C_{1} d \overline{I_{1}} / d \nu\right)+\bar{Z}\left(C_{2} d \overline{I_{2}} / d \nu\right)$, where $\bar{Z}$ is the weighted average of the negative Zeeman coefficients, with the weights being $\mid$ R.I $_{i} \mid$. The fits for $C_{1}$ and $C_{2}$ are $C_{1}=-0.15 \pm 2.25 \mathrm{mG}$ and $C_{2}=-3.57 \pm 3.22 \mathrm{mG}$, which are consistent with the values obtained for $c_{1}$ and $c_{2}$ from the simultaneous Zeeman fit to individual $V$ spectra mentioned above.

The Zeeman Stokes $V$ signal due to a field of a few hundred $\mu$ Gauss is small (i.e., a line broadening of a few hundred $\mathrm{Hz}$ ), and the noise level in the spectra needs to be sufficiently low in order to obtain at least a $2 \sigma$ detection. We developed simulations for the observed $\mathrm{CN}$ lines with different noise levels and magnetic field strengths, to estimate the remaining integration time required to obtain a detection. Crutcher et al. (1999) obtained a value of $B_{\text {los }} \simeq 0.75 \mathrm{mG}$ for one of the velocity components of $\mathrm{DR} 21(\mathrm{OH})$ by observing the $N=1 \rightarrow 0$ transition of $\mathrm{CN}$, which is associated with a critical density $n_{c} \approx 10^{5} \mathrm{~cm}^{-3}$. Assuming that the magnetic field strength varies with $n^{1 / 2}$, where $n$ is the the average gas density of the observed region, we should expect that the magnetic field we probe with our observations will be somewhat stronger. Since $n_{c} \approx 10^{6} \mathrm{~cm}^{-3}$ at the $N=2 \rightarrow 1$ transition, $B_{\text {los }}$ should be at most a few times stronger than the value obtained with the $N=1 \rightarrow 0$ transition. Our simulations show that for a $2 \mathrm{mG}$ field, we need to have our noise level down to $\simeq 5 \mathrm{mK}$ to be able to get at least a $2 \sigma$ detection. The RMS noise in our 
data is currently $\simeq 16 \mathrm{mK}$, implying that we need more observations to obtain a credible detection. Assuming an average system temperature of $400 \mathrm{~K}$, with a bandwidth of $61 \mathrm{kHz}$, we will require about 48 more hours of on-source integration time. This time estimate is comparable with the observing time that Crutcher et al. (1999) spent on OMC1n and DR21OH to obtain a Zeeman detection in the $\mathrm{CN}(N=1 \rightarrow 0)$ transition.

\section{Summary}

We recently designed and successfully commissioned a Four-Stokes-Parameter spectral line Polarimeter (FSPPol) at the CSO in November 2008. The simple design of FSPPol does not contain any mirrors or grids to redirect or split the radiation beam, and the instrument is conveniently mounted in the elevation tube between the tertiary mirror behind the telescope dish and the Nasmyth focus where the heterodyne receiver is located. FSPPol transmits the beam to the receiver through half-wave and quarter-wave plates that are presently optimized for observations at 226 $\mathrm{GHz}$.

We used FSPPol for linear and circular polarization measurements in the spectral lines of interstellar molecules during the months of July, September and October 2009. We measured a linear polarization level of $\simeq 1 \%$ to $2.5 \%$ due to the Goldreich-Kylafis effect in the spectral line wings of ${ }^{12} \mathrm{CO}(J=2 \rightarrow 1)$ in Orion KL/IRc2, and our results are consistent with previous observations (Girart et al. 2004). We also started Zeeman observations on the $N=2 \rightarrow 1$ transition of CN in $\mathrm{DR} 21(\mathrm{OH})$ for the first time. At this point we have obtained about 10 hours of on-source integration time, and our preliminary data analysis shows that although we have not detected a Zeeman signal in the $\mathrm{CN}(N=2 \rightarrow 1)$ lines, the overall contribution from the instrumental effects in the Stokes $V$ spectrum is low. Further observations of $\mathrm{CN}(N=2 \rightarrow 1)$ are ongoing for this source and other star forming regions.

The authors thank B. Dalrymple, S. H. Chen and T. Officer for their help in the development of FSPPoL, H. Shinnaga for helping with the Zeeman calculations and M. Azimlu and S. Chitsazzadeh for assistance during observations. M. H.'s research is funded through the NSERC Discovery Grant, Canada Research Chair, Canada Foundation for Innovation, Ontario Innovation Trust, and Western's Academic Development Fund programs. The Caltech Submillimeter Observa-

tory is funded through the NSF grants AST 08-38261 and AST 05-40882 to the California Institute of Technology.

\section{REFERENCES}

Afsar, M., N. 1987, IEEE: Transactions on Instrumentation and Measurement, 36, 2, 554

Birch et al. 1994, IEEE: Transactions on Microwave Theory and Techniques, 42, 6, 956 
Crutcher, R. M., Troland, T. H., Goodman, A. A., Heiles, C., Kazes, I., Myers, P. C. 1993, ApJ, 407, no. 1 , p. 175

Crutcher, R. M., Troland, T. H., Lazareff, B., \& Kazès, I. 1996, ApJ, 456, 217

Crutcher, R. M., Troland, T. H., Lazareff, B., Paubert, G. \& Kazès, I. 1999, ApJ, 514, 2, L121

Deguchi, S. and Watson, W. D. 1984, ApJ, 285, 126

Falgarone, E., Troland, T. H., Crutcher, R. M. \& Paubert, G. 2008, A\&A, 487, 1, 247

Forbrich, J., Wiesemeyer, H., Thum, C., Belloche, A. and Menten, K. M. 2008, A\&A, 492, 757

Girart, J.M., Greaves, J.S., Crutcher, R.M. \& Lai, S.-P. 2004, Ap\&SS, 292, 119

Goldreich, P. \& Kylafis, N., D 1981, ApJ, 243 L75

Greaves, J. S.; Holland, W. S.; Jenness, T.; Chrysostomou, A.; Berry, D. S.; Murray, A. G.; Tamura, M.; Robson, E. I.; Ade, P. A. R.; Nartallo, R.; Stevens, J. A.; Momose, M.; Morino, J.-I.; Moriarty-Schieven, G.; Gannaway, F.; Haynes, C. V. 2003, MNRAS, 340, 2, 353

Heiles, C. 2001, PASP, 113, 788, 1243

Heiles, C., Perillat, P., Nolan, M., Lorimer, D., Bhat, R., Ghosh, T., Howell, E., Lewis, M., O’Neil, K., Salter, C. and Stanimirovic, S. 2001a, PASP, 113, 788, 1247

Heiles, C., Perillat, P., Nolan, M., Lorimer, D., Bhat, R., Ghosh, T., Lewis, M., O’Neil, K., Salter, C. and Stanimirovic, S. 2001b, PASP, 113, 788, 1274

Hildebrand, R. H., Dotson, J L., Dowell, C. D., Novak, G., Schleuning, D A. \&Vaillancourt, J. 1997, Publ. Astron. Soc. Pac., 109, 307

Hildebrand, R. H., Dotson, J. L., Dowell, C. D., Schleuning, D. A. \& Vaillancourt, J. E. 1999, ApJ, $516,2,834$

Hildebrand, R. H., Kirby, L., Dotson, J. L., Houde, M. and Vaillancourt, J. E. 2009, ApJ, 696, 567

Houde, M., Vaillancourt, J. E., Hildebrand, R. H., Chitsazzadeh, S. and Kirby, L. 2009, ApJ, 706, 1504

Li, H., Dowell, C. D., Kirby, L., Novak, G. \& Vaillancourt, J. E. 2008, Applied Optics IP, 47, 3, 422

Magalhaes, A. M., Rodrigues, C. V., Margoniner, V. E., Pereyra, A., \& Heathcote, S. 1996, in Polarimetry of the Interstellar Medium, ASP Conf. Ser., 97, 118

Marrone, D., P. \& Rao, R. 2008, Proceedings of the SPIE, 7020, 70202B

Plante, R. L., Lo, K. Y. and Crutcher, R., M. 1995, ApJ, 445, L113 
Platt, S. R., Hildebrand, R. H., Pernic, R. J., Davidson, J. A. \& Novak, G. 1991, PASP, 103, p. 1193

Sarma, A. P. and Momjian, E. 2009, ApJ, 705, L176

Sarma, A. P., Troland, T. H. and Romney, J. D 2001, ApJ, 554, L217

Savini, G., Ade, P. A. R., House, J., Pisano, G., Haynes, V. and Bastien, P. 2009, Applied Optics, 48, 11, 2006

Shinnaga et al. 2010, in preperation

Shinnaga, H., Tsuboi, M \& Kasuga, T. 1999, PASJ, 51, 175

Thum, C., Wiesemeyer, H., Paubert, G., Navarro, S. and Morris, D. 2008, PASP, 120, 869, 777

Troland, T. H. and Crutcher, R., M. 2008, ApJ, 680, 457

Wiktorowicz, S. J. \& Matthews, K. 2008, PASP, 120, 874, 1282 


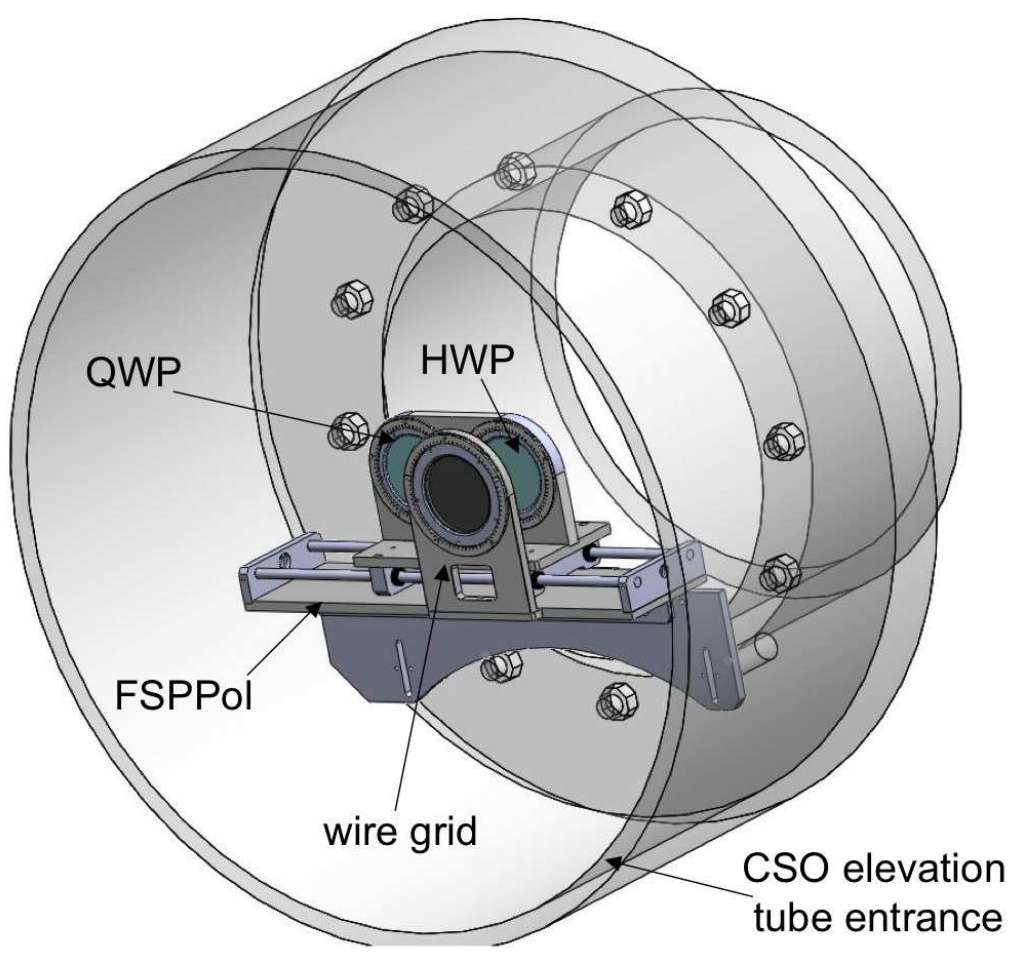

Fig. 1. - The schematic diagram of FSPPol installed in the elevation tube at the CSO. The polarizing grid, used for testing, at the entrance of FSPPol is also shown. 


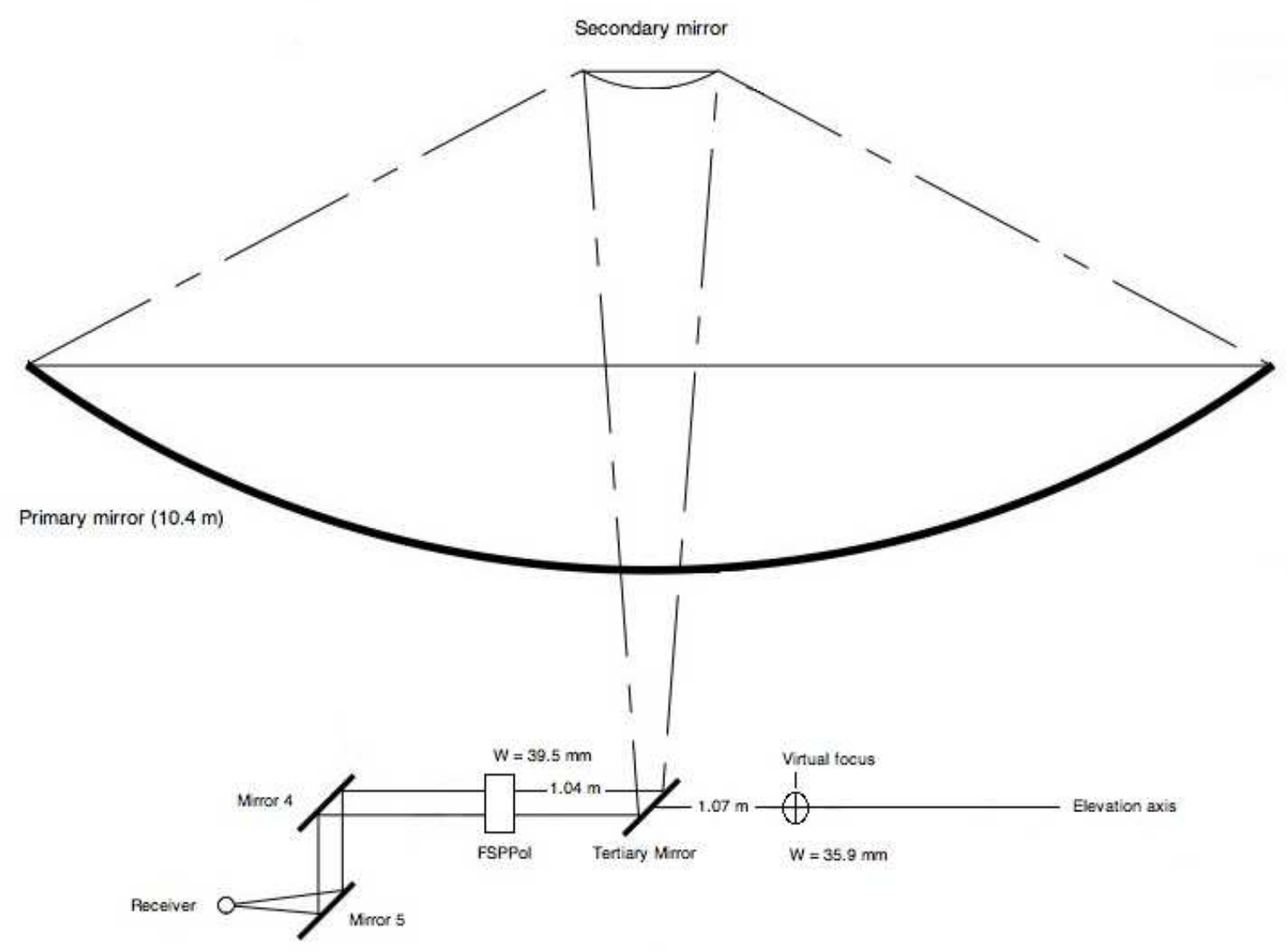

Fig. 2. - The schematic diagram of the CSO telescope, also showing the position of FSPPol at $1.04 \mathrm{~m}$ from the tertiary mirror towards Mirror 4. The telescope has an alt-azimuth mount and the receiver is located at one of the two Nasmyth foci. The virtual focus of the tertiary mirror is located $1.07 \mathrm{~m}$ behind the tertiary, and the beam waists $(\mathrm{W})$ at the virtual focus and at FSPPol are $35.9 \mathrm{~mm}$ and $39.5 \mathrm{~mm}$, respectively. 


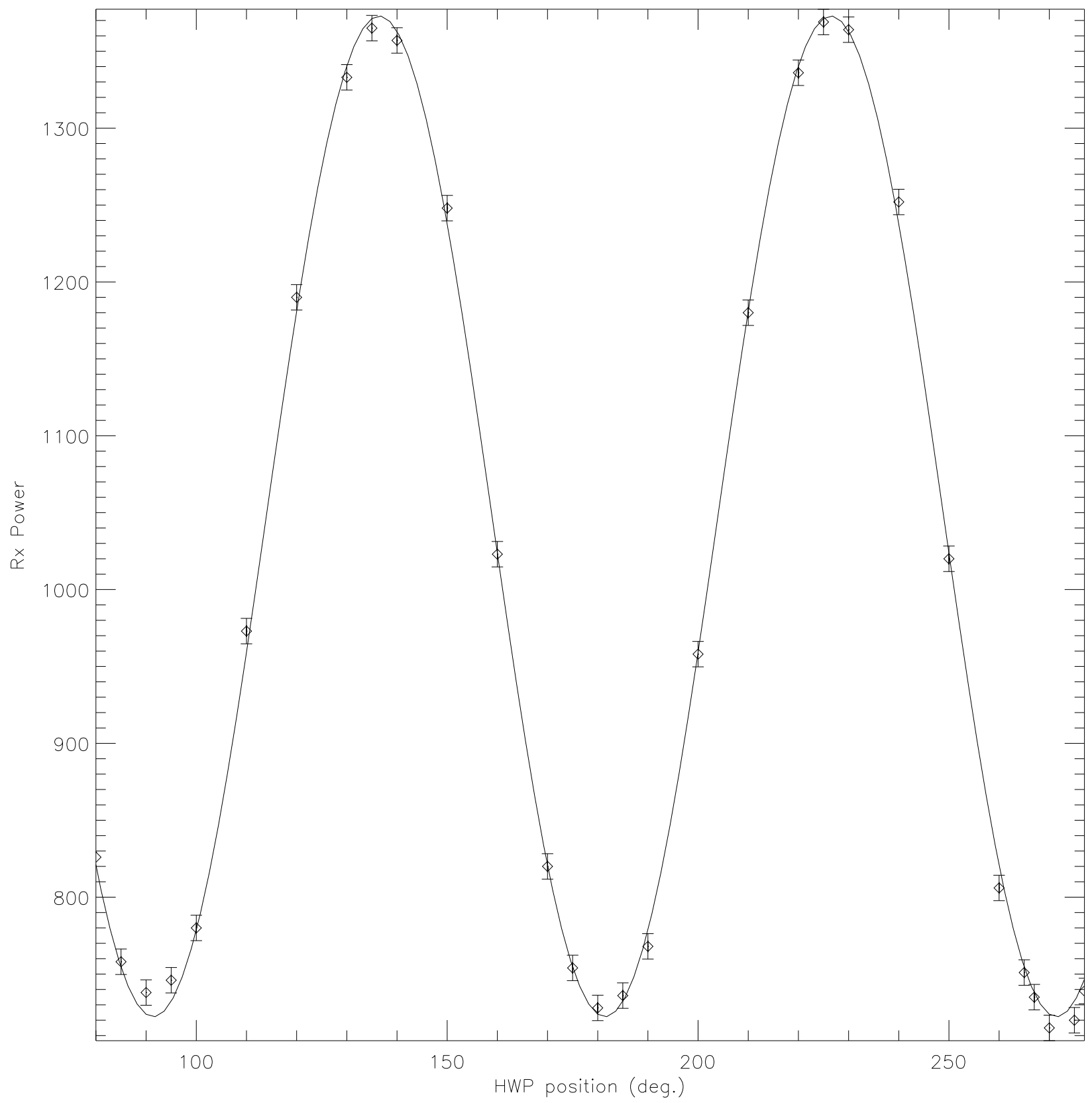

Fig. 3. - The graph of the variation of the power measured from a cold load with the CSO receiver against different orientations of the HWP with respect to the receiver polarization axis. The $R_{x}$ power is in arbitrary units. 


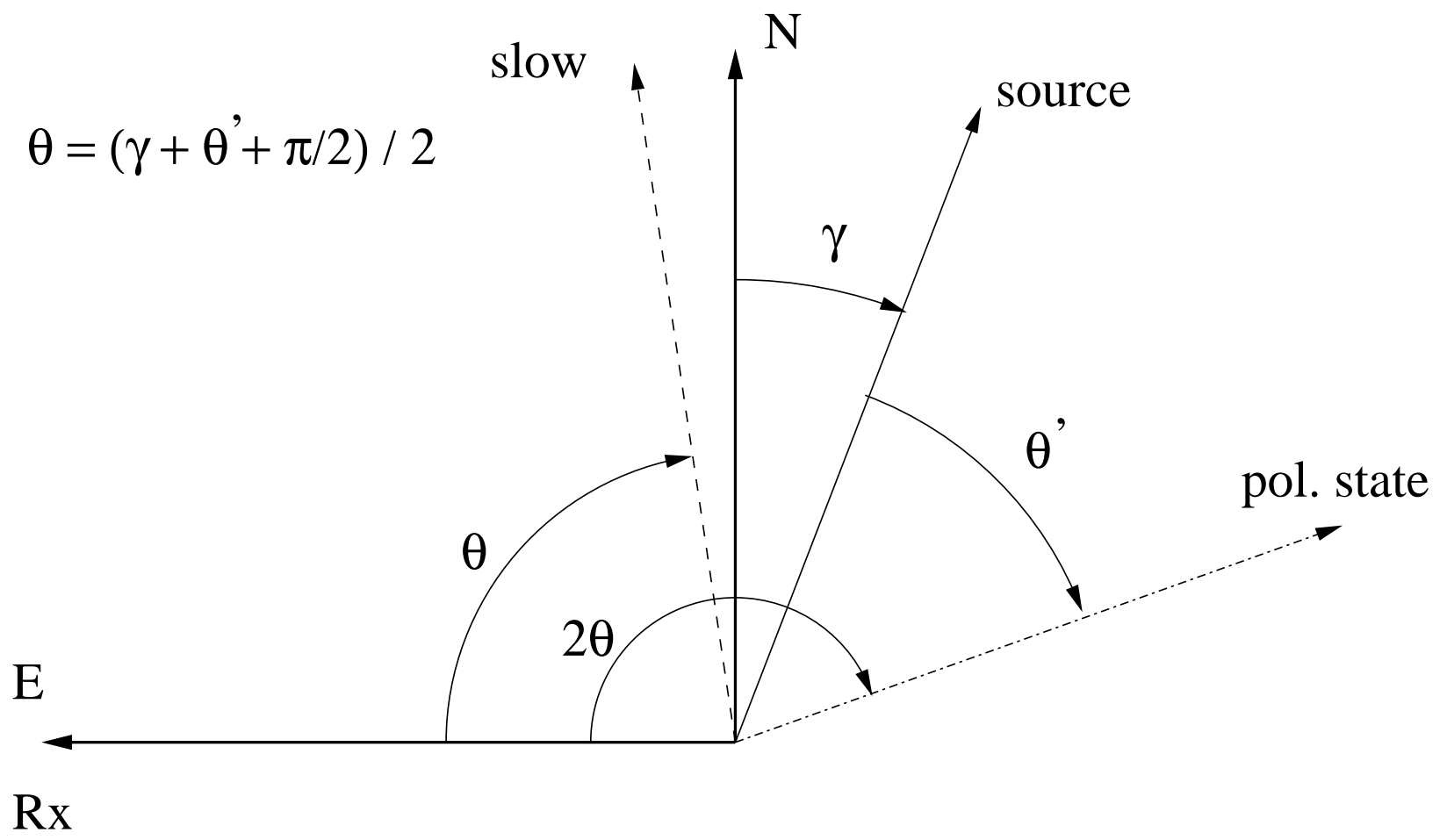

Fig. 4.- A schematic diagram for our linear polarization measurements. The equatorial coordinate system shows North and East on the sky. The receiver's polarization axis $\left(\mathrm{R}_{x}\right)$ projected on the sky in the equatorial system, lies along the East-West sky axis, $\theta$ is the angle between $\mathrm{R}_{x}$ and the slow axis of the HWP (denoted by "slow"), $\gamma$ is the parallactic angle of the source, and $\theta^{\prime}$ the angle at which we want to measure the polarization state in the frame of the source. Accordingly, we have $\theta=\left(\gamma+\theta^{\prime}+90^{\circ}\right) / 2$. 

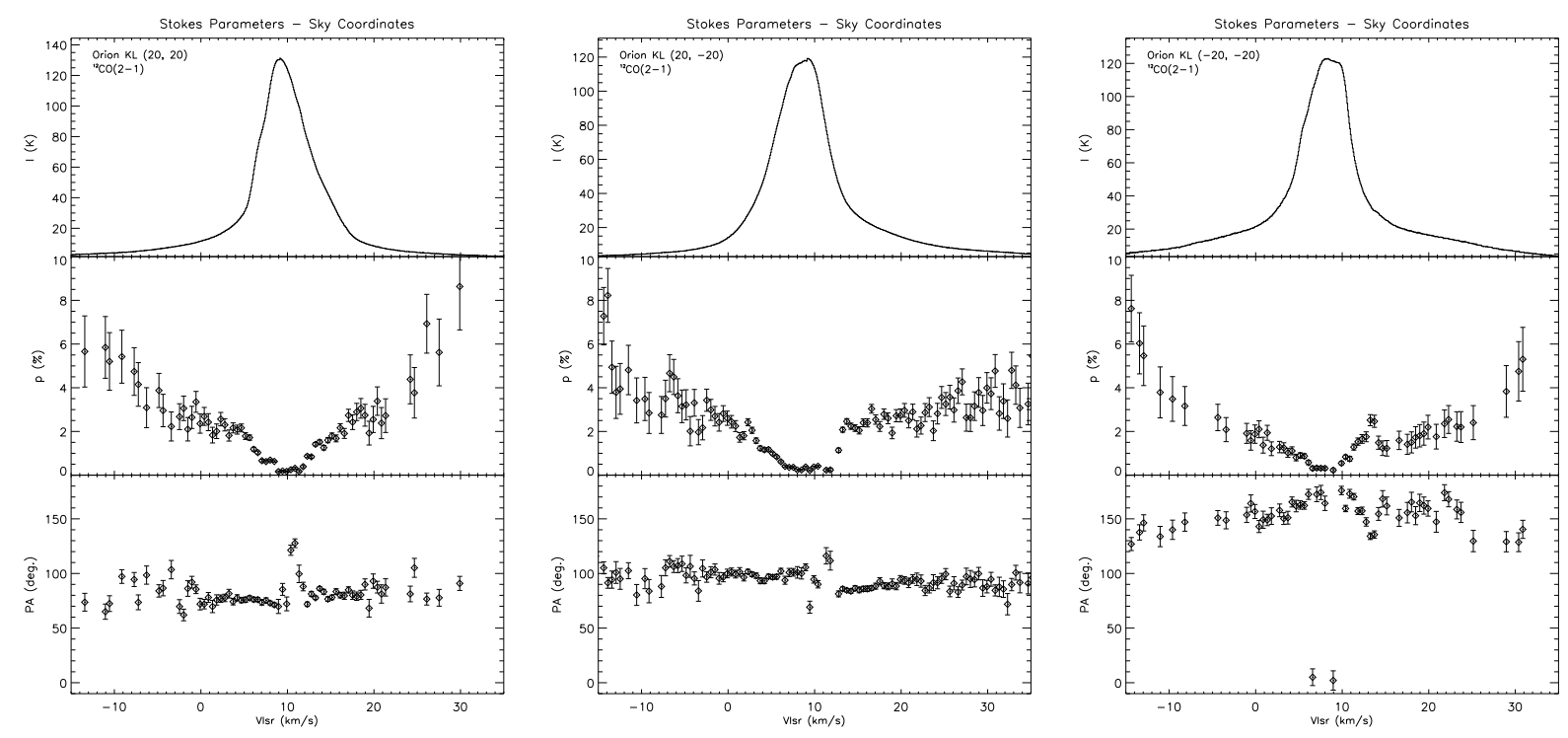

Fig. 5.- Linear polarization measurements of ${ }^{12} \mathrm{CO}(J=2 \rightarrow 1)$ in Orion KL/IRc2; (top) Stokes $I$, corrected for the telescope beam efficiency; (middle) The polarization level $p$ and (bottom) angles PA across the spectral line. 


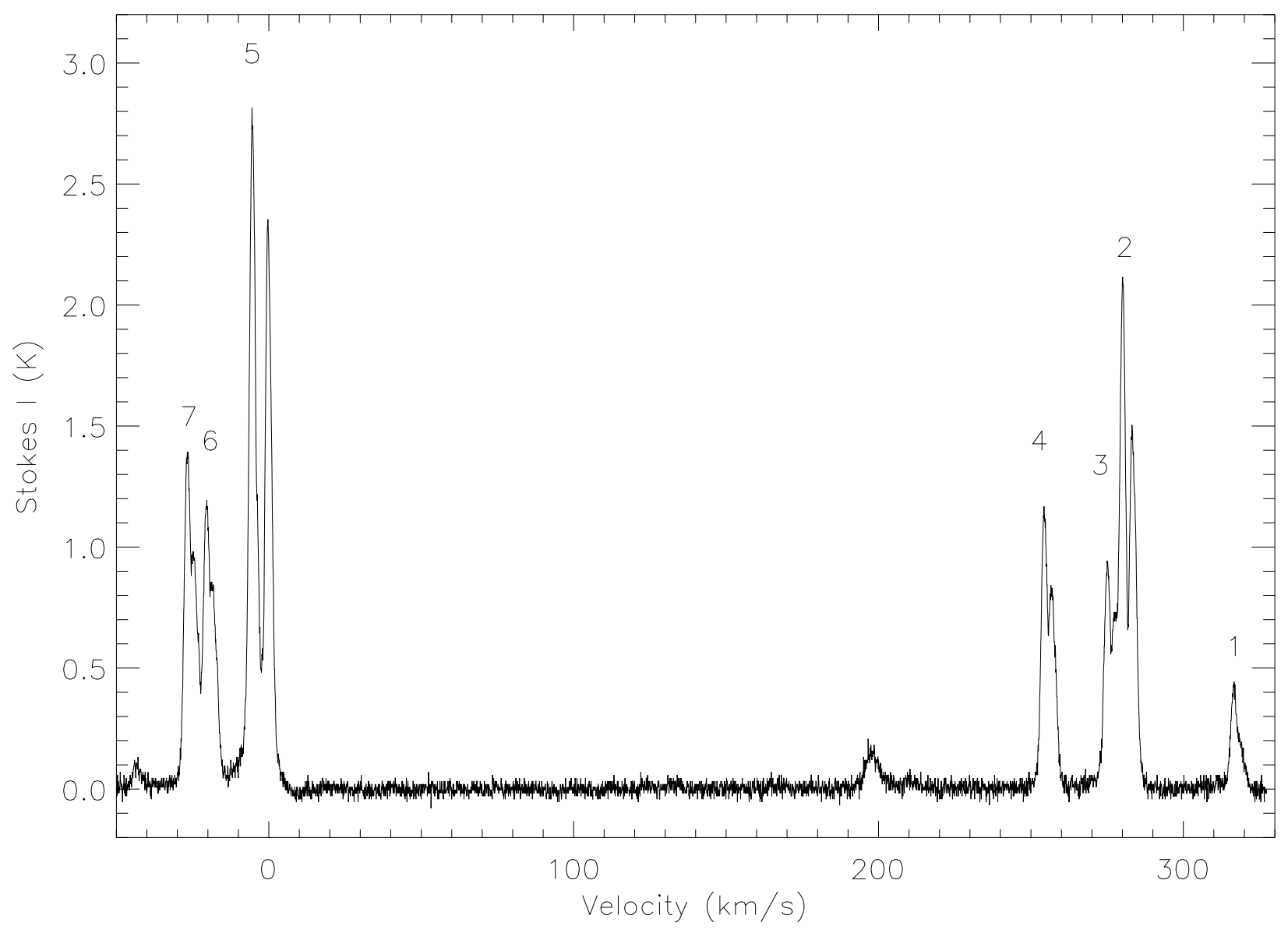

Fig. 6.- Stokes $I$ spectrum of the nine strong hyperfine components of $\mathrm{CN}(N=2 \rightarrow 1)$ towards $\mathrm{DR} 21(\mathrm{OH})$. Line 5 is actually three blended lines that appear as one. 

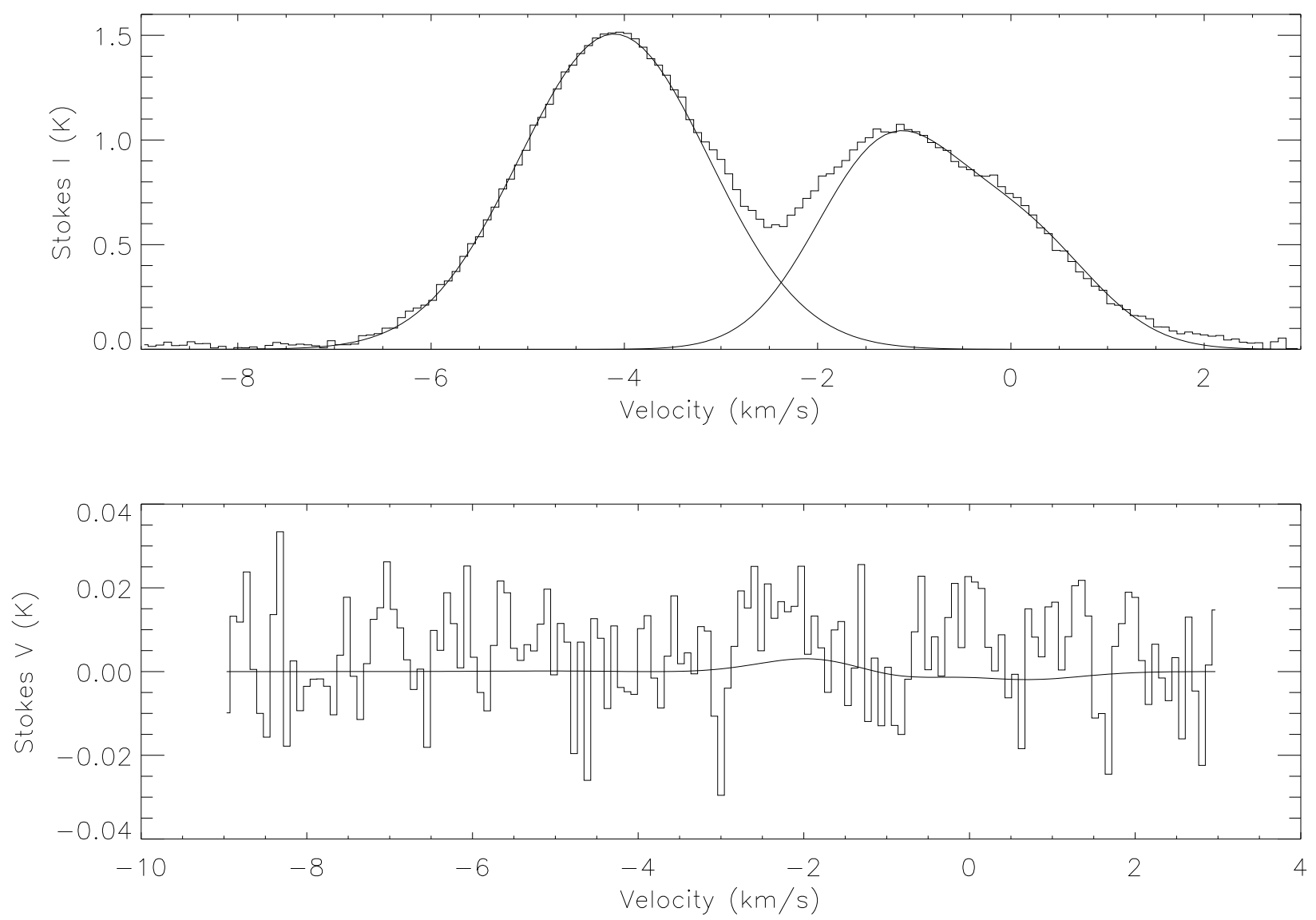

Fig. 7.- (top) The averaged Stokes $I$ spectrum for the CN $(N=2 \rightarrow 1)$ hyperfine lines $1,2 \& 4$ in DR21(OH) that have negative Zeeman coefficients. The solid lines are the Gaussian fits to the two velocity components of Stokes $I$ spectrum. (bottom) The averaged Stokes $V$ spectrum for the same hyperfine lines. The solid line is the fit for the Zeeman expression to the Stokes $V$ spectrum, with instrumental effects removed. 
Table 1. Properties of the wave plates

\begin{tabular}{ll}
\hline \hline \multicolumn{1}{c}{ Property } & \\
& \\
\hline Material & single crystal Quartz \\
Indices of refraction: & \\
Ordinary & $2.106 \pm 0.006^{\mathrm{a}}$ \\
Extraordinary & $2.154 \pm 0.007^{\mathrm{a}}$ \\
Thickness & $13.82 \mathrm{~mm}(\mathrm{HWP}), 6.90 \mathrm{~mm}(\mathrm{QWP})$ \\
Anti-reflection coating: & \\
Material & High density Polypropylene (HDPP) \\
\hline
\end{tabular}

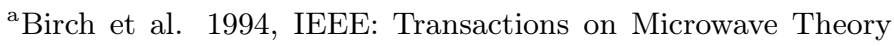
and Techniques, 42, 6, 956 
Table 2. Polarization measurements on $\mathrm{CO} J=2 \rightarrow 1$ in Orion KL/IRc2

\begin{tabular}{cccccrr}
\hline \hline & \multicolumn{3}{c}{$p(\%)$} & & \multicolumn{2}{c}{ PA (deg) } \\
\cline { 2 - 3 } Offsets $(\operatorname{arcsec})^{\mathrm{a}}$ & Blue $^{\mathrm{b}}$ wing & Line center & Red $^{\mathrm{c}}$ wing & & Blue $^{\mathrm{b}}$ wing & Red $^{\mathrm{c}}$ wing \\
\hline$\left(20^{\prime \prime}, 20^{\prime \prime}\right)$ & $2.1 \pm 0.3$ & $0.35 \pm 0.04$ & $1.8 \pm 0.2$ & & $77.1 \pm 4.2$ & $81.2 \pm 3.4$ \\
$\left(20^{\prime \prime},-20^{\prime \prime}\right)$ & $1.6 \pm 0.2$ & $0.29 \pm 0.04$ & $2.4 \pm 0.2$ & & $97.8 \pm 3.3$ & $88.1 \pm 2.9$ \\
$\left(-20^{\prime \prime},-20^{\prime \prime}\right)$ & $1.1 \pm 0.3$ & $0.29 \pm 0.07$ & $1.5 \pm 0.4$ & & $154.8 \pm 7.1$ & $155.6 \pm 8.1$ \\
\hline
\end{tabular}

${ }^{\mathrm{a}}$ Offsets are with respect to $\operatorname{IRc} 2\left(\alpha=05^{\mathrm{h}} 35^{\mathrm{m}} 14.5^{\mathrm{s}}, \delta=-05^{\circ} 22^{\prime} 30.4^{\prime \prime} ; \mathrm{J} 2000.0\right)$.

${ }^{\mathrm{b}} p$ and PA are averaged over the velocity range of -4 to $6 \mathrm{~km} \mathrm{~s}^{-1}$ (see Figure 5 ).

${ }^{\mathrm{c}} p$ and $\mathrm{PA}$ are averaged over the velocity range of 13 to $23 \mathrm{~km} \mathrm{~s}^{-1}$. 
Table 3. Hyperfine components of $\mathrm{CN}(N=2 \rightarrow 1)^{\mathrm{a}}$

\begin{tabular}{cccccc}
\hline \hline Line & $(N, J, F) \rightarrow\left(N^{\prime}, J^{\prime}, F^{\prime}\right)$ & $\nu(\mathrm{MHz})$ & $Z(\mathrm{~Hz} / \mu \mathrm{G})$ & ${\mathrm{R} . \mathrm{I}^{\mathrm{b}}}$ & $|Z \times \mathrm{R} . \mathrm{I}|^{\mathrm{c}}$ \\
& & & & & \\
\hline 1 & $(2,3 / 2,3 / 2) \rightarrow(1,1 / 2,3 / 2)$ & 226632.19 & -0.72241 & 0.59259 & 0.42809 \\
2 & $(2,3 / 2,5 / 2) \rightarrow(1,1 / 2,3 / 2)$ & 226659.58 & -0.70995 & 2.0 & 1.41991 \\
3 & $(2,3 / 2,1 / 2) \rightarrow(1,-1 / 2,1 / 2)$ & 226663.70 & 0.62277 & 0.59259 & 0.36905 \\
4 & $(2,3 / 2,3 / 2) \rightarrow(1,1 / 2,1 / 2)$ & 226679.38 & -1.18326 & 0.74074 & 0.87649 \\
5 & $(2,5 / 2,5 / 2) \rightarrow(1,3 / 2,3 / 2)$ & 226874.17 & 0.70995 & 2.016 & 1.43127 \\
5 & $(2,5 / 2,7 / 2) \rightarrow(1,3 / 2,5 / 2)$ & 226874.75 & 0.40035 & 3.200 & 1.28112 \\
5 & $(2,5 / 2,3 / 2) \rightarrow(1,3 / 2,1 / 2)$ & 226875.90 & 1.18326 & 1.200 & 1.41991 \\
6 & $(2,5 / 2,3 / 2) \rightarrow(1,3 / 2,3 / 2)$ & 226887.35 & 1.46973 & 0.3840 & 0.56438 \\
7 & $(2,5 / 2,5 / 2) \rightarrow(1,3 / 2,5 / 2)$ & 226892.12 & 1.05692 & 0.3840 & 0.40586 \\
\hline
\end{tabular}

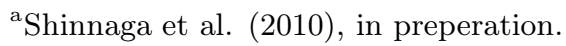

${ }^{\mathrm{b}}$ Relative intensity.

${ }^{\mathrm{c}}$ Relative sensitivity to $B_{\text {los }}$. 